\title{
Modeling and Mitigating 5G Wireless Downlink Interferences for Low-altitude Aerial vehicles
}

\author{
Wenbo Wang*, Thilo Fath ${ }^{\dagger}$, Mikko Valkama*, and Elena Simona Lohan* \\ * Electrical Engineering Unit, Tampere University, Finland, Emails: firstname.lastname@tuni.fi \\ $\dagger$ Airbus, Germany
}

\begin{abstract}
Future 5G networks will serve both terrestrial and aerial users, thanks to their network slicing and flexible numerology capabilities. The probability of Line-of-Sight $(\mathrm{LoS})$ propagation will be intuitively higher for aerial users than for terrestrial users and this will provide a trade-off between increased capacity and increased interference. Our paper analyzes theoretically this trade-off and proposes solutions based on downlink multiantenna beamforming and joint optimization of the signal-tointerference ratio of multiple aerial users. It is shown that Multiple-Input-Single-Output solutions offer the most convenient tradeoff between complexity and capacity/interference performance. Simulation results are provided for $\mathbf{m m W a v e}$ bands and low-altitude aerial vehicles.
\end{abstract}

Index Terms-autonomous aerial vehicles, drones, interference, communication links, Signal-to-Interference Ratio (SIR), Multiple-Input-Single-Output (MISO)

\section{INTRODUCTION AND MOTIVATION}

$5 \mathrm{G}$ cellular communications have already become a reality. The $5 \mathrm{G}$ network are meant to serve a multitude of users/robots/devices and will offer a multitude of services, thanks to the new paradigms introduced in $5 \mathrm{G}$, such as network slicing [1, 2, 3, 4], network virtualization [1, 5, 6], and Software-Defined Network (SDN) [7, 8]. Future 5G wireless communications will serve not only terrestrial users, but also aerial users, such as Unmanned Aerial Aircraft (UAV), popularly known as drones, and other low-altitude aircraft (e.g., flying taxis, flying emergency aircraft, crops surveillance aircraft, etc.). The altitude's effect on wireless communication links is still not fully understood, especially when referring to altitudes of few $\mathrm{km}$. The low-altitude terminology used in our title and in our work refers to aircraft altitudes up to 3 $\mathrm{km}$, corresponding mostly to uncontrolled airspace, such as U-space aerial space [9] and G-class users [10] in aviation community.

Another differentiating factor between terrestrial and aerial users/devices is the fact that there is an increased likelihood of LoS connectivity between a terrestrial 5G Base Station (BS) and an aerial user compared to the situation when the user is on the ground. The increased LoS probability can increase reliability of the wireless connectivity, by ensuring a better Received Signal Strength (RSS) and a better Signal-to-Noise Ratio (SNR) than in Non Line-of-Sight (NLoS) scenarios. At the same time, it can also increase the amount of interference from non-desired transmitters (i.e., transmitters which are not transmitting useful information to the aerial user), and thus it may decrease the Signal-to-Interference Ratio (SIR).
The tradeoff between increased RSS and increased interference has been previously studied in the context of terrestrial users, for example, in [11] for cooperative beamforming with massive Multiple-Input Multiple-Output (mMIMO) solutions, in [12] for full-duplex solutions in Cloud Radio Access Networks (C-RAN), or in [13] for ultra dense terrestrial small cell deployments. The interference for downlink transmissions towards aerial users has been recently studied in [14] with focus on UAVs with maximum altitude of $300 \mathrm{~m}$.

Our paper novelty is two-folds, namely: i) we provide a SIR model for aerial users with altitudes up to $3 \mathrm{~km}$, extrapolating the 3GPP aerial channel modeling which are currently limited to $300 \mathrm{~m}$ altitudes, and by including also cloud attenuation modeling, under eight different scenarios and ii) we propose an interference mitigation approach based on Multiple-Input Single-Output (MISO) and MIMO solutions.

\section{Channel loss modeling}

Wireless channel losses between an aerial receiver and a ground transmitter, including path losses and other atmospheric losses, have a large influence on determining the achievable SIR of the receivers. As we consider low-altitude vehicles with an altitude of up to $3000 \mathrm{~m}$, we consider several hypotheses:

1. above $300 \mathrm{~m}$ altitude, we assume we have Free Space Loss (FSL) and LoS condition. This hypothesis is based on two observations:

a. [15] reported that above $504 \mathrm{~m}$, in near-urban and sub-urban areas, the measurements of path loss at L-band (i.e., $968 \mathrm{MHz}$ ) and C-band (i.e., $5060 \mathrm{MHz}$ ) fit the free space loss well;

b. Rural Macrocell (RMa) and Urban Macrocell (UMa) models in [16] converge to the free space loss model at $300 \mathrm{~m}$ altitude.

2. below $300 \mathrm{~m}$ altitude, we use the model based on 3GPP rural macrocell (RMa) and urban macrocell (UMa) channel path-loss models [16]. In addition, according to 3GPP channel models, above $40 \mathrm{~m}$ and $100 \mathrm{~m}$ altitude in rural and urban, signals propagate purely in LoS condition, while below these altitude values, we have a combination of LoS and NLoS with probabilities defined in [16].

3. Signal attenuation due to propagation through clouds is also included in our modeling for aerial users; our model is based on ITU recommendations [17]. 
With these hypotheses in mind, the overall channel loss $\overline{P L}$ (large-scale loss, in $\mathrm{dB}$ scale) is given by,

$$
\overline{P L}= \begin{cases}L^{3 \mathrm{GPP}}+L_{\mathrm{cl}}\left(d_{\mathrm{cl}}\right), & h_{u} \leq 300 \\ L_{300}^{3 \mathrm{GPP}}+20 \log _{10}\left(\frac{d}{d_{300}}\right)++L_{\mathrm{cl}}\left(d_{\mathrm{cl}}\right), & h_{u}>300\end{cases}
$$

where $L^{3 \mathrm{GPP}}$ is the $3 \mathrm{GPP}$ channel loss model in [16], $L_{\mathrm{cl}}(\cdot)$ is the clouds attenuation model in [17], $d_{\mathrm{cl}}$ (in meter) is the propagation distance within clouds, $L_{300}^{3 G P P}$ is a constant calculated by the above mentioned 3GPP channel loss model at $300 \mathrm{~m}$ altitude, $d_{300}$ (in meter) is the transmitter-receiver (T-R) separation distance when the receiver is at $300 \mathrm{~m}$ altitude, $d$ (in meter) is the actual T-R separation distance, $h_{u}$ (in meter) is the altitude of users. The above parameters are also depicted in Fig. 1 for clarity purposes.

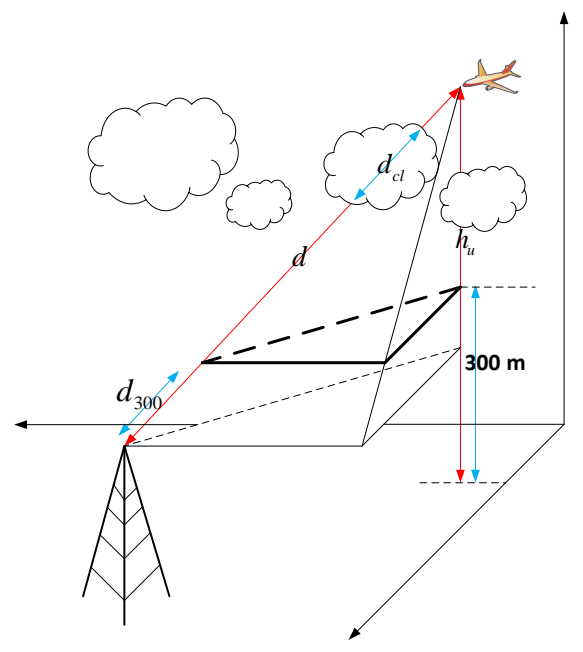

Fig. 1: Example of the channel loss model for aerial users.

\section{INTERFERENCE MODELS}

Cellular networks have been traditionally optimized to offer best performance to terrestrial users, e.g., by maximizing their SIR. SIR maximization can come with an increase in the TX power, and thus with an increase in the interference towards other users sharing the same frequency bands. In particular, such interference can be highly detrimental to aerial users, which are more likely to be in LoS connections than terrestrial ones. In order to model and analyze the interference, we will focus on two cases:

1) Multiple transmitters (TX) and single receiver (RX), i.e., the non-cooperative case. In this situation, each RX aims at maximizing its own SIR independently, no coordination of transmitters exists;

2) Multiple transmitters (TX) and multiple receivers (RX), i.e., the cooperative case. The difference to the previous case is that now, the designer is trying to maximize the overall performance for all users (terrestrial and aerial), by defining a properly chosen utility function which depends on the individual SIR.

In addition to the two cases described above, we will also consider the following four sub-cases:

i. Single-Input-Single-Output (SISO), i.e., omni-directional antennas both at TX and RX side;

ii. Single-Input-Multiple-Output (SIMO), i.e., omnidirectional antenna at TX side and directional/multi-array antenna at RX side;

iii. MISO, i.e., directional/multi-array antenna at TX side and omni-directional antenna at RX side and

iv. MIMO, i.e., directional/multi-array antenna both at TX and RX sides.

The research questions we ask next are:

- to what extent are aerial users more affected by interference compared to terrestrial users, assuming that all other conditions are unchanged?

- which of the four above-mentioned antenna sub-cases is to be chosen by a designer who wants to achieve the best trade-off between interference and SIR and what metric is to be used for this?

\section{A. Multiple TX and single $R X$}

Let us consider first the non-cooperative scenario depicted in Fig. 2a that consists of $N$ transmitters (TX) and one receiver (RX). The numbers of transmitters form a set denoted by $\mathcal{N}_{t x}=\{1,2,3, \cdots, N\}$. We make the assumption that the desired signal is from the $k$-th transmitter, while the other $N-1$ transmitters only cause interference. The numbers of transmitters whose signals reach the receiver simultaneously is a subset of the set $\mathcal{N}_{t x}$; this subset is denoted by $\mathcal{K}_{t}$ and we know that $\mathcal{K}_{t x} \subseteq \mathcal{N}_{t x}$. The SIR is defined as,

$$
\mathrm{SIR}=\frac{P_{r}}{P_{\text {in }}}
$$

where SIR is in linear scale, $P_{r}$ is the received signal power (in Watt) from the desired transmitter, $P_{i n}$ is the overall received interference signal power (in Watt) from all interfering base stations.

By slightly modifying the Friis formula, $P_{r}$ yields to,

$$
P_{r}=P_{t}^{(k)} G_{t}^{(k)} \xi_{t}^{(k)} G_{r}^{(k)} \xi_{r} L^{(k)}
$$

where $P_{t}^{(k)}$ is the transmitted signal power (in Watt) from the $k$-th transmitter, $G_{t}^{(k)}$ is the $k$-th transmitter's gain (linear scale), $\xi_{t}^{(k)}$ is the loss (linear scale) in the $k$-th transmitter, $G_{r}^{(k)}$ is the receiver gain (linear scale) when receiving signals from the $k$-th transmitter, $\xi_{r}$ is the loss (linear scale) in the receiver, e.g., due to feeders, $L^{(k)}$ is the path-loss (linear scale) of the channel between the $k$-th transmitter and the receiver. The channel loss $L^{(k)}$ is proportional to the T-R separation distance and the carrier signal frequency, according to the selected path-loss channel model.

Similarly, the overall received interference signal power $P_{\text {in }}$ yields to, 


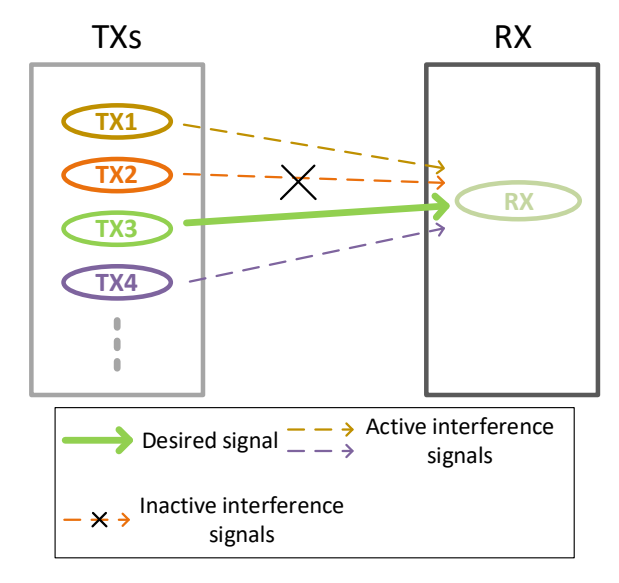

(a) Illustration of a scenario with multiple TX and one RX.

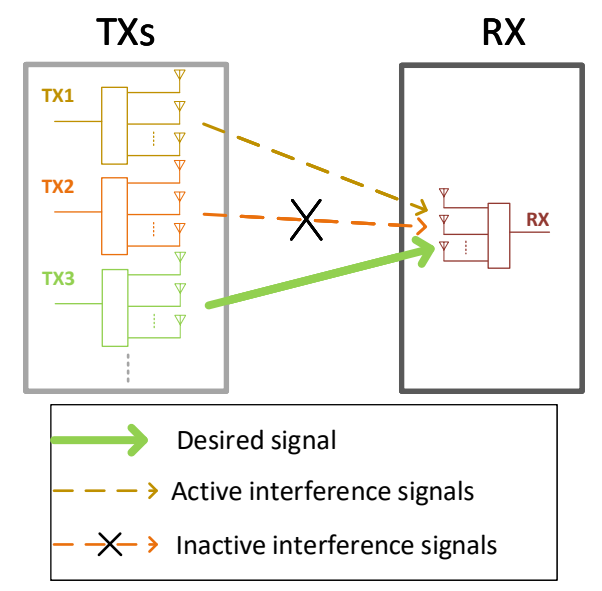

(b) Illustration of interference channels in MIMO systems.

Fig. 2: Interference channels in multiple TX and one RX scenario.

$$
P_{\text {in }}=\sum_{i \in \mathcal{K}_{t x}, i \neq k} P_{t}^{(i)} G_{t}^{(i)} \xi_{t}^{(i)} G_{r}^{(i)} \xi_{r} L^{(i)}
$$

Under (3) and (4), (2) becomes,

$$
\mathrm{SIR}=\frac{P_{t}^{(k)} G_{t}^{(k)} \xi_{t}^{(k)} G_{r}^{(k)} L^{(k)}}{\sum_{i \in \mathcal{K}_{t x}, i \neq k} P_{t}^{(i)} G_{t}^{(i)} \xi_{t}^{(i)} G_{r}^{(i)} L^{(i)}}
$$

the loss $\xi_{r}$ in the receiver is treated as temporally and spatially invariant hence can be cancelled, whereas the receiver gain $G_{r}^{(i)}$ varies in the multiple antennas system.

1) SISO: SISO refers to the case with an omni-directional antenna at both the transmitter and receiver side. The SISO system in multiple TX and single RX scenario is the benchmark for the other cases considered in this paper. In the SISO system, if we assume that the gain and loss in all the transmitters are identical, the SIR in (5) is now,

$$
\operatorname{SIR}_{\mathrm{SISO}}=\frac{P_{t}^{(k)} L^{(k)}}{\sum_{i \in \mathcal{K}_{t x}, i \neq k} P_{t}^{(i)} L^{(i)}}
$$

Within a given dynamic range, the transmitted power $P_{t}$ typically has constraints (e.g., physical limitation of amplifiers, domestic regulatory, economic considerations etc.), and thus the channel loss $L$ dominates the SIR.

2) MISO/SIMO: MISO refers to the case with multiple antennas at the transmitter side and an omni-directional antenna at the receiver side. SIMO refers to the case with an omnidirectional antenna at the transmitter side and multiple antennas at the receiver side.

In the MISO system, if we assume that losses for all transmitters are identical, the SIR in (5) transforms into,

$$
\operatorname{SIR}_{\mathrm{MISO}}=\frac{P_{t}^{(k)}\left[\mathbf{L}^{(k)}\right]^{\mathrm{H}} \mathbf{G}_{t}^{(k)}}{\sum_{i \in \mathcal{K}_{t x}, i \neq k} P_{t}^{(i)}\left[\mathbf{L}^{(i)}\right]^{\mathrm{H}} \mathbf{G}_{t}^{(i)}}
$$

where $[\cdot]^{\mathrm{H}}$ is Hermitian transpose, $\mathbf{L}^{(k)}$ is $n_{t} \times 1$ channel loss vector (linear scale) from the $k$ th transmitter, $\mathbf{G}_{t}^{(k)}$ is $n_{t} \times 1$ gain vector (linear scale) of the $k$ th transmitter, $n_{t}$ denotes the number of antennas in the transmitter.

In the SIMO system, if we assume the gain and loss in all transmitters are identical, the SIR in (5) becomes,

$$
\operatorname{SIR}_{\mathrm{SIMO}}=\frac{P_{t}^{(k)}\left[\mathbf{G}_{r}\right]^{\mathrm{H}} \mathbf{L}^{(k)}}{\sum_{i \in \mathcal{K}_{t x}, i \neq k} P_{t}^{(i)}\left[\mathbf{G}_{r}\right]^{\mathrm{H}} \mathbf{L}^{(i)}}
$$

where $\mathbf{G}_{r}$ is the $n_{r} \times 1$ gain vector (linear scale) of the receiver, $\mathbf{L}^{(k)}$ is $n_{r} \times 1$ channel loss vector (linear scale) from the $k$-th transmitter with $n_{r}$ denoting the number of antennas at the receiver.

3) MIMO: MIMO refers to the case with multiple antennas at both the transmitter and receiver side. In the MIMO system, if we assume the losses of all transmitters are identical, the SIR in (5) is then,

$$
\operatorname{SIR}_{\text {MIMO }}=\frac{P_{t}^{(k)}\left[\mathbf{G}_{r}\right]^{\mathrm{H}} \mathbf{L}^{(k)} \mathbf{G}_{t}^{(k)}}{\sum_{i \in \mathcal{K}_{t x}, i \neq k} P_{t}^{(i)}\left[\mathbf{G}_{r}\right]^{\mathrm{H}} \mathbf{L}^{(i)} \mathbf{G}_{t}^{(i)}}
$$

where $\mathbf{L}^{(k)}$ is the $n_{r} \times n_{t}$ channel loss vector (linear scale) from the $k$-th transmitter.

\section{B. Multiple TX and multiple RX}

Let us now consider the cooperative scenario shown in Fig. 3 that consists of $N$ transmitters (TX) and $M$ receivers (RX). In order to keep consistency of the notations used in this paper, we define the numbers of transmitters as a set $\mathcal{N}_{t x}=\{1,2,3, \cdots, N\}$ and the numbers of receivers as a set $\mathcal{M}_{r x}=\{1,2,3, \cdots, M\}$. The desired signal is transmitted from the $k$-th transmitter to the $j$-th receiver. The numbers of transmitters whose signals reach the $j$-th receiver simultaneously is a subset of the set $\mathcal{N}_{t x}$ denoted by $\mathcal{K}_{t x}^{(j)}$ with 
$\mathcal{K}_{t x}^{(j)} \subseteq \mathcal{N}_{t x}$. The numbers of receivers receiving signals at the same time is a subset of the set $\mathcal{M}_{r x}$ denoted by $\mathcal{J}_{r x}$ with $\mathcal{J}_{r x} \subseteq \mathcal{M}_{r x}$.

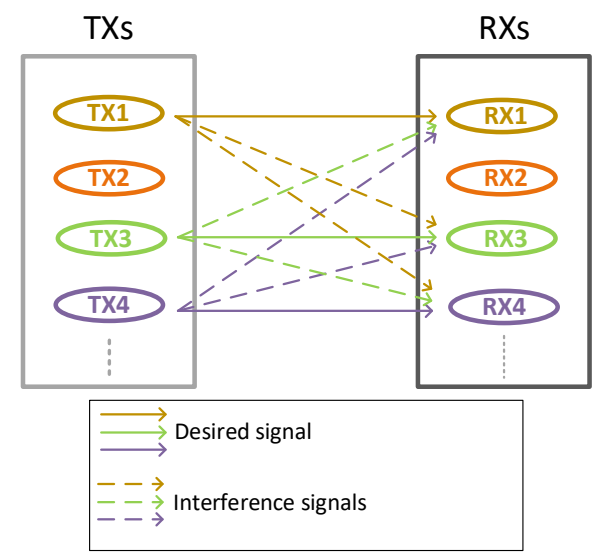

Fig. 3: Illustration of a scenario with multiple TX and multiple RX.

The SIR in (5) at $j$-th receiver is then,

$$
\operatorname{SIR}^{(j)}=\frac{P_{t}^{(k j)} G_{t}^{(k j)} \xi_{t}^{(k)} G_{r}^{(k j)} L^{(k j)}}{\sum_{i \in \mathcal{K}_{t x}^{(j)}, i \neq k} P_{t}^{(i j)} G_{t}^{(i j)} \xi_{t}^{(i)} G_{r}^{(i j)} L^{(i j)}}
$$

where $P_{t}^{(k j)}$ is the transmitted power (in Watt) from the $k$-th transmitter to the $j$-th receiver, $G_{t}^{(k j)}$ is the antenna gain (linear scale) from the $k$-th transmitter to the $j$-th receiver, $G_{r}^{(k j)}$ is the antenna gain (linear scale) of the $j$-th receiver, $L^{(k j)}$ is the channel loss (linear scale) from the $k$-th transmitter to the $j$-th receiver.

At a time instant, the SIR of all active receivers could be represented by a set,

$$
\mathcal{S}=\left\{\operatorname{SIR}^{(j)}: j \in \mathcal{J}_{r x}\right\}
$$

In order to analyze the overall performance of multiple receivers scenarios, it is common to apply utility functions to the above SIR set [18]. In this work, it is of interest minimize the SIR for aerial users, i.e., to find first the minimum value in the set $\mathcal{S}$. Hence we define a utility function $U$ as,

$$
\begin{aligned}
& U: \operatorname{SIR}^{(j)} \mapsto \operatorname{SIR}_{\text {min }}, \\
& \operatorname{SIR}^{(j)}, \operatorname{SIR}_{\text {min }} \in \mathcal{S}, \\
& U\left(\operatorname{SIR}^{(j)}\right)=\min _{j \in \mathcal{J}_{r}} \operatorname{SIR}^{(j)} .
\end{aligned}
$$

Besides, for terrestrial users, weighted sum-rate as a utility function is commonly used [18],

$$
U^{W S R}\left(\operatorname{SIR}^{(j)}\right)=\frac{1}{|\mathcal{S}|} \sum_{j \in \mathcal{J}_{r}} \operatorname{SIR}^{(j)}
$$

In this paper, since it is of our interest to investigate how the aerial users are affected by the interference, the lowest SIR of an aerial user needs to be above a certain threshold, we select (12) as our metric to evaluate the level of interference in multiple TX and multiple RX scenarios.

1) SISO: In the SISO system, if we assume that the gains and losses of all the transmitters are identical, the SIR in (10) becomes,

$$
\operatorname{SIR}_{\text {SISO }}^{(j)}=\frac{P_{t}^{(k j)} L^{(k j)}}{\sum_{i \in \mathcal{K}_{t x}^{(j)}, i \neq k} P_{t}^{(i j)} L^{(i j)}}
$$

2) MISO/SIMO: In the MISO system, if we assume that the losses of all transmitters are identical, the SIR in (10) is then,

$$
\operatorname{SIR}_{\text {MISO }}^{(j)}=\frac{P_{t}^{(k j)}\left[\mathbf{L}^{(k j)}\right]^{\mathrm{H}} \mathbf{G}_{t}^{(k j)}}{\sum_{i \in \mathcal{K}_{t x}, i \neq k} P_{t}^{(i j)}\left[\mathbf{L}^{(i j)}\right]^{\mathrm{H}} \mathbf{G}_{t}^{(i j)}}
$$

In the SIMO system, if we assume that the gains and losses of all transmitters are identical, the SIR in (10) becomes,

$$
\operatorname{SIR}_{\text {SIMO }}^{(j)}=\frac{P_{t}^{(k j)}\left[\mathbf{G}_{r}^{(k j)}\right]^{\mathrm{H}} \mathbf{L}^{(k j)}}{\sum_{i \in \mathcal{K}_{t x}, i \neq k} P_{t}^{(i j)}\left[\mathbf{G}_{r}^{(i j)}\right]^{\mathrm{H}} \mathbf{L}^{(i j)}}
$$

3) MIMO: In the MIMO system, if we assume that the losses of all transmitters are identical, the SIR in (10) transforms to,

$$
\operatorname{SIR}_{\text {MIMO }}^{(j)}=\frac{P_{t}^{(k j)}\left[\mathbf{G}_{r}^{(k j)}\right]^{\mathrm{H}} \mathbf{L}^{(k j)} \mathbf{G}_{t}^{(k j)}}{\sum_{i \in \mathcal{K}_{t x}, i \neq k} P_{t}^{(i j)}\left[\mathbf{G}_{r}^{(i j)}\right]^{\mathrm{H}} \mathbf{L}^{(i j)} \mathbf{G}_{t}^{(i j)}}
$$

\section{INTERFERENCE FOR AERIAL USERS}

\section{A. Aerial users vs terrestrial users}

As we discussed in the introduction, on the one hand, the desired signals received by aerial users undergo LoS propagation, which means lower signal attenuation compared to NLoS propagation for the same channel; on the other hand, the interfering signals experience also LoS conditions, and thus are subject to lower attenuation as well. In contrast, both desired and the interfering signals likely experience a mixture of LoS and NLoS loss for terrestrial users. Intuitively, it is difficult to give a simple judgment, whether aerial users are more vulnerable to the interference or not compared to terrestrial users.

Using (6) and assuming that the transmitted power of all transmitters is the same (i.e., no power control techniques are applied) and that the T-R separation distance for both aerial users and terrestrial users is the same, we numerically compare SIR for terrestrial and aerial users.

Fig. 4 demonstrates the comparison of SIR for an aerial and a terrestrial user. The considered carrier frequency is $30 \mathrm{GHz}$, the altitude of the aerial user is $150 \mathrm{~m}$, the altitude of the terrestrial user is $2 \mathrm{~m}$, the height of all transmitters is $35 \mathrm{~m}$, the $\mathrm{T}-\mathrm{R}$ separation distance between the desired transmitter and the respective user is $200 \mathrm{~m}$, the T-R separation distance between the interfering transmitters and the user follows a uniform distribution $\mathcal{U}(250,5000)$ (in meter). Due to the relatively low 


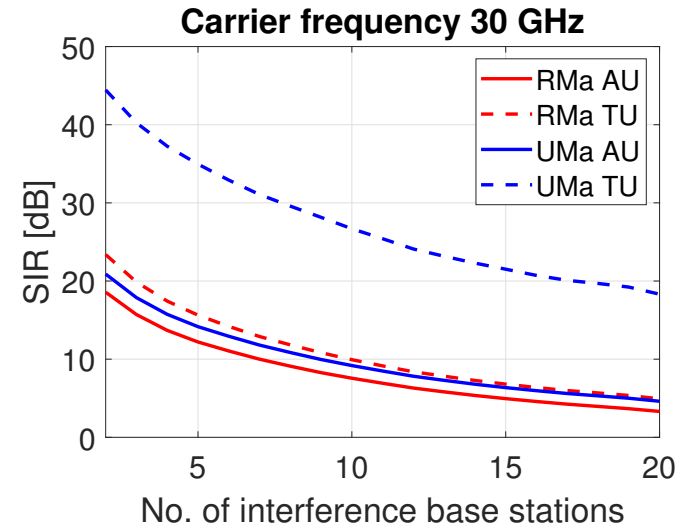

Fig. 4: Comparisons of SIR for an aerial user (AU) and a terrestrial user (TU).

altitude, the attenuation caused by clouds is not considered in this evaluation.

Using (14), in addition to the assumptions made in Fig. 4, we implement numerical analysis considering the attenuation caused by clouds. Fig. 5 shows the comparison of SIR for three aerial and three terrestrial users. The T-R separation distance between the desired transmitter and three aerial and three terrestrial users is $170,200,230 \mathrm{~m}$ respectively. The considered attenuation caused by clouds is, under consideration of $0.5 \mathrm{~g} / \mathrm{m}^{3}$ liquid water density, the ratio between the propagation distance within clouds and the T-R separation distance which follows a uniform distribution $\mathcal{U}(0,0.5)$. The SIR shown in Fig. 5 is under the utility function (12) for three aerial/terrestrial users.

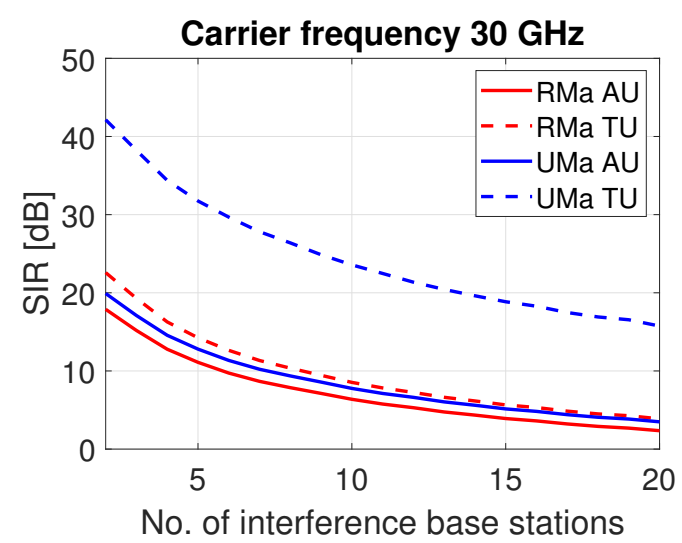

Fig. 5: Comparisons of SIR for 3 aerial users (AU) and 3 terrestrial users (TU).

Clearly, in both Fig. 4 and Fig. 5, the aerial users are more vulnerable to interference than terrestrial users. Especially in the urban area, the SIR of terrestrial users is at least $10 \mathrm{~dB}$ higher than the SIR of aerial users.

\section{Multi-Antenna-BASEd InTERFEREnCE Mitigation}

Ideally, by applying multiple antenna systems (i.e., MISO, SIMO, MIMO), the interference could be cancelled by appropriately using the null parts in the antenna radiation pattern.
However, a perfect cancellation of interference has very high demands on the system, for example w.r.t. perfect beam alignment. In wireless communications for aerial drones, it is sensible to consider the aggregate interference to be small. For example, in (7) the interference can be considered to be

$$
\sum_{i \in \mathcal{K}_{t x}, i \neq k} P_{t}^{(i)}\left[\mathbf{L}^{(i)}\right]^{\mathrm{H}} \mathbf{G}_{t}^{(i)}=\varepsilon
$$

where $\varepsilon$ is a small positive number.

Utilization of multiple-antenna drone systems is considered in the $5 \mathrm{G}$ standard. It is thus of interest to see how the MISO/SIMO (i.e., considering the downlink as MISO scenario and the uplink as SIMO scenario) system could mitigate interference in wireless links for aerial drones.

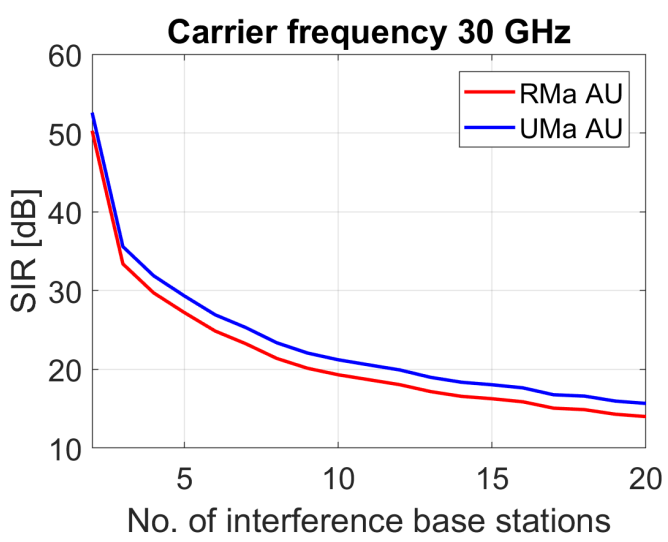

Fig. 6: SIR of an aerial user (AU) applying a MISO system.

In Fig. 6, besides of considering a $8 \times 8$ URA (Uniform Rectangular Array) in the transmitters, we used the same parameters as in Fig. 4, while the misalignment of beams is considered in Fig. 6. In comparison to Fig. 4, it can be seen that the MISO system significantly suppresses the interference in both RMa AU and UMa AU. For instance, for the case of 20 interference source transmitters, the MISO system improves the SIR by around $10 \mathrm{~dB}$ compared to the SISO system.

In the future, considering the upcoming mmWave bands, it is very promising to apply multiple-antenna systems on board of drones. We also simulate the MIMO scenario to have an idea how much the on-board multiple antenna system could help to mitigate interference.

Comparing Fig. 7 with Fig. 6, we can observe that due to $4 \times 4$ URA on board, the SIR is generally improved by $2-3 \mathrm{~dB}$. However, the benefit of $2-3 \mathrm{~dB}$ in SIR performance is gained at the cost of higher complexity for all aerial users' on-board communication systems.

\section{DESIGN RECOMMENDATIONS}

Based on the presented results, the design recommendations for using $5 \mathrm{G}$ to support communication data links for lowaltitude aerial vehicles can be summarized as follows:

- The use of antenna arrays at the base station (i.e., MISO configuration) significantly reduces inference for the signals of aerial users. 


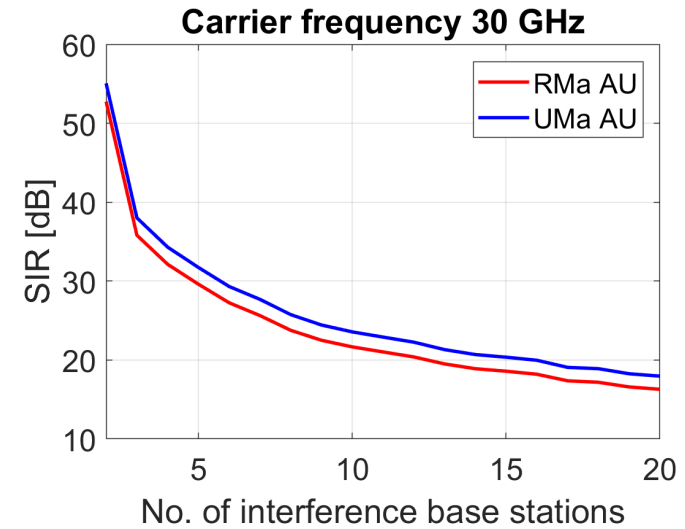

Fig. 7: SIR of an aerial user (AU) applying a MIMO system.

- The use of MIMO solutions will provide only a few $\mathrm{dB}$ extra gain for the receiver compared to the MISO case, at the cost of additional complexity due to the antenna arrays needed on-board of the aircraft. Therefore, MISO solutions are recommended as best tradeoff between complexity and performance. In addition, as future $5 \mathrm{G}$ base stations are to be equipped with multiple-antenna systems, MISO solutions are fully feasible in the near term future.

\section{CONCLUSION}

Future 5G networks have large potential in serving aerial users. In contrast to terrestrial users, for which traditional communication networks are optimized for, aerial users are in LoS propagation conditions for most of the time (e.g., during cruising phase). Through numerical analysis, it has been shown that aerial users are more vulnerable to interference than terrestrial users. By applying a MISO/SIMO system (i.e., applying a multiple antenna system at the base station), a large improvement in the mitigation of interference can been achieved. The MIMO system does not bring too much extra improvement compared to the MISO/SIMO system but largely increases complexity of the on-board communication system.

\section{ACKNOWLEDGEMENTS}

The authors would like to thank the Academy of Finland (ULTRA project), for funding this research work.

\section{REFERENCES}

[1] D. A. Chekired, M. A. Togou, L. Khoukhi, and A. Ksentini, "5gslicing-enabled scalable sdn core network: Toward an ultra-low latency of autonomous driving service," IEEE Journal on Selected Areas in Communications, vol. 37, no. 8, pp. 1769-1782, Aug. 2019. DOI: 10.1109/JSAC.2019.2927065.

[2] X. Foukas, G. Patounas, A. Elmokashfi, and M. K. Marina, "Network slicing in 5g: Survey and challenges," IEEE Communications Magazine, vol. 55, no. 5, pp. 94-100, May 2017. DOI: 10.1109/MCOM.2017. 1600951
[3] I. Afolabi, T. Taleb, K. Samdanis, A. Ksentini, and H. Flinck, "Network slicing and softwarization: A survey on principles, enabling technologies, and solutions," IEEE Communications Surveys Tutorials, vol. 20, no. 3 , pp. 2429-2453, 2018. DOI: 10.1109/COMST.2018.2815638.

[4] F. D'Ursol, C. Grasso, C. Santoro, F. F. Santoro, and G. Schembra, "The tactile internet for the flight control of uav flocks," in 20184 th IEEE Conference on Network Softwarization and Workshops (NetSoft), Jun. 2018, pp. 470-475. DOI: 10.1109/NETSOFT.2018.8458493.

[5] X. Zhang and Q. Zhu, "Scalable virtualization and offloading-based software-defined architecture for heterogeneous statistical qos provisioning over $5 \mathrm{~g}$ multimedia mobile wireless networks," IEEE Journal on Selected Areas in Communications, vol. 36, no. 12, pp. 2787-2804, Dec. 2018. DOI: 10.1109/JSAC.2018.2871327.

[6] A. N. Al-Quzweeni, A. Q. Lawey, T. E. H. Elgorashi, and J. M. H. Elmirghani, "Optimized energy aware 5g network function virtualization," IEEE Access, vol. 7, pp. 44 939-44958, 2019. DOI: 10.1109/ ACCESS.2019.2907798.

[7] I. Bor-Yaliniz and H. Yanikomeroglu, "The new frontier in ran heterogeneity: Multi-tier drone-cells," IEEE Communications Magazine, vol. 54, no. 11, pp. 48-55, Nov. 2016. DOI: 10.1109/MCOM.2016. $1600178 \mathrm{CM}$.

[8] W. F. Elsadek, "Toward hyper interconnected iot world using sdn overlay network for ngn seamless mobility," in 2016 IEEE International Conference on Cloud Computing Technology and Science (CloudCom), Dec. 2016, pp. 460-463. DOI: 10.1109/CloudCom.2016.0078.

[9] L. Legros, R. Garrity, and A. Hately, Initial view on principles for the u-space architecture, https://www.sesarju.eu/node/3402 (accessed Aug 2019), 2019.

[10] Airspace management operations manual - procedures for flexible use of airspace, https://www.traficom.fi/sites/default/files/media/regulation/ASMtoimintaka\%CC\%88sikirja\%20v1.8\%20en.pdf (accessed Aug 2019), 2019.

[11] R. Dong, A. Li, W. Hardjawana, Y. Li, X. Ge, and B. Vucetic, "Joint beamforming and user association scheme for full-dimension massive mimo networks," IEEE Transactions on Vehicular Technology, vol. 68, no. 8, pp. 7733-7746, Aug. 2019. DOI: 10.1109/TVT.2019.2923415.

[12] C. Fang, P. Li, and K. Feng, "Joint interference cancellation and resource allocation for full-duplex cloud radio access networks," IEEE Transactions on Wireless Communications, vol. 18, no. 6, pp. 3019-3033, Jun. 2019. DOI: 10.1109/TWC.2019.2908173.

[13] J. Xiao, C. Yang, A. Anpalagan, Q. Ni, and M. Guizani, "Joint interference management in ultra-dense small-cell networks: A multi-domain coordination perspective," IEEE Transactions on Communications, vol. 66, no. 11, pp. 5470-5481, Nov. 2018. DOI: 10.1109/TCOMM. 2018.2851215 .

[14] V. Yajnanarayana, Eric Wang, Y. -P, S. Gao, S. Muruganathan, and $X$. Lin Ericsson, "Interference mitigation methods for unmanned aerial vehicles served by cellular networks," in 2018 IEEE $5 G$ World Forum (5GWF), Jul. 2018, pp. 118-122. DOI: 10.1109/5GWF.2018.8517087.

[15] D. W. Matolak and R. Sun, "Air-ground channel characterization for unmanned aircraft systems-Part III: The suburban and near-urban environments," IEEE Transactions on Vehicular Technology, vol. 66, no. 8, pp. 6607-6618, 2017.

[16] 3GPP - 3rd Generation Partnership Project; Technical Specification Group Radio Access Network; Study on enhanced LTE support for aerial vehicles (release 15), 3GPP TR 36.777 V15.0.0, http://www.3gpp.org/DynaReport/36-series.htm, Jan. 2018.

[17] ITU, Attenuation due to clouds and fog, Recommendation ITU-R P.8407, Dec. 2017

[18] Y. Liu, Y. Dai, and Z. Luo, "Coordinated Beamforming for MISO Interference Channel: Complexity Analysis and Efficient Algorithms," IEEE Transactions on Signal Processing, vol. 59, no. 3, pp. 1142-1157, Mar. 2011. DOI: $10.1109 /$ TSP.2010.2092772. 